\title{
La incidencia de la violencia y la criminalidad en la localización sectorial de las
}

empresas en el occidente de México*

\section{The incidence of violence and crime in sectorial business}

location in western Mexico

José Luis Hernández Angeles**

Recibido: 13 de marzo de 2019

Revisado: 3 de abril de 2019

Aprobado: 10 de junio de 2019 


\section{Resumen}

La localización y concentración de la actividad económica responde a muchos factores - algunos de ellos positivos y otros negativos - que desde la perspectiva de la teoría económica provocan economías de aglomeración. Uno de los elementos desfavorecedores y que provocan externalidades negativas son el crimen y la violencia (Hampley \& McPheters, 1975; Pichardo-Muñíz \& Chavarría, 2016). En particular, aquellos que afectan directa e indirectamente a las empresas son los analizados en el presente estudio. Los resultados obtenidos que aplican técnicas de análisis espacial y econometría lineal indican que, para el occidente de México, la afectación por la delincuencia oficialmente reportada es dispar y altamente dependiente de los sectores económicos analizados. No obstante, en la mayoría de los casos, factores que generan externalidades positivas, como el nivel educativo, son significativamente relevantes y acordes a los planteamientos propuestos.

Palabras clave: concentración económica, economía espacial, crimen y economía.

Clasificación JEL: D62, R1 1, R12.

\section{Abstract}

The localization and concentration of the economic units depends on many factors - some of which are positive and others negative - that under the umbrella of the neoclassical economic theory get to form agglomeration economies. One of the unfavorable elements which that tend to create negative externalities are crime and violence (Hampley \& McPheters, 1975; Pichardo-Muñíz \& Chavarría, 2016). Particularly those affecting businesses directly and indirectly are the ones analyzed in this study. The obtained results applying spatial statistics and econometric techniques suggest that for Western Mexico the effects of crime and violence are not equivalently distributed among all the formal economic sector, hence specific cases should be analyzed. Nevertheless, other positive factors such as the average educational level are found to be relevant and going accordingly to the proposed hypothesis.

Keywords: economic concentration, spatial economics, crime and economics.

Classification JEL: D62, R11, R12. 


\section{Introducción}

La concentración y crecimiento de un sistema económico depende de muchos factores que se traducen en condiciones que permiten su funcionamiento apropiado y eficiente. Entre estos se encuentran factores generales que corresponden a la composición demográfica, de profesionalización o nivel educativo, mecanismos de regulación, confianza institucional y participación ciudadana, y la instauración de un estado de derecho (IMCO, 2007; Mendoza, 2014). Por otra parte, también se encuentran elementos de corte particular y que se vinculan directamente a la toma de decisión de las empresas de dónde localizarse (Mugellini, 2013).

Uno de estos factores es la calidad del entorno social, visto mediante el clima de la seguridad pública existente por la incidencia y prevalencia delictivas. Las anteriores, abordadas desde la perspectiva de la teoría económica, se consideran como externalidades negativas (Hamley \& McPheters, 1975), pues tanto la violencia como el crimen afectan a la actividad económica formal e informal al imponer mayores costos a la producción y aumentar la incertidumbre, dadas potenciales fallas en el sistema institucional existente (IMCO, 2007; Mendoza, 2014). Todo lo anterior, finalmente, se traduce en afectaciones al nivel de ingreso, la producción bruta y la calidad de vida de la población afectada (Goulas \& Zervoyianni, 2015).

A pesar de ser un fenómeno de alta relevancia social, el análisis de los efectos que tiene la criminalidad en la localización de la actividad económica ha sido estudiada escuetamente. Entre los esfuerzos hechos para conocer más profundamente el fenómeno, en México, el Instituto Nacional de Geografía y Estadística (Inegi) comenzó a levantar la Encuesta Nacional de Victimización de Empresas (ENVE) de manera bienal (Inegi, 2016). Esta encuesta ha ayudado a estimar la cifra negra y el costo del delito - entre otras cosas - que afectan particularmente el sector productivo del país. En asociación a las implicaciones que tiene la delincuencia y la criminalidad en las actividades económicas, trabajos como el de Detotto y Otranto (2010) para Italia o los de Ruiz-Estrada y Ndoma $(2014,2016)$ para Guatemala y Centroamérica demuestran la existencia de una afectación negativa, bien sea en el producto interno bruto o en la tasa de crecimiento de país analizado.

Por otra parte, existe una serie de trabajos de análisis particulares que se pueden tomar como referente. Entre estos podemos encontrar el caso de González Andrade (2014) en el que se analizan los efectos en el crecimiento económico de México a nivel estatal, donde descubre que, aunque hay efectos mínimos, estos son generales para todos los delitos analizados - homicidios, lesiones y robos - y van acordes a los planteamientos teóricos que señalan efectos negativos. Por otra parte, el estudio de Enamorado, LópezCalva y Rodríguez-Castelán (2014) complementa los resultados anteriores al analizar cómo los homicidios por la guerra contra el narcotráfico afectan el comportamiento del 
ingreso per cápita registrado en México entre 2005 y 2010. En este caso, encuentra que las afectaciones no son concluyentes salvo que se observe el fenómeno a nivel municipal por tasa poblacional ( $0.20 \%$ por D.E.) o por el grado de urbanización presentada $(0.13 \%$ por D.E. para municipios urbanizados y $0.19 \%$ para semiurbanos).

Otros trabajos que se abocan a estudiar concretamente la localización económica son los de Sloan, Cuadill y Mixon, Jr. (2016), en donde se analiza cómo la incidencia delictiva violenta afecta la apertura de nuevos restaurantes, para el primer caso; o el de Matti y Ross (2016), que evalúa cómo la ocurrencia de estos delitos afecta los esfuerzos de los emprendedores por establecerse de manera formal tomando en consideración políticas paliativas, como lo son la atracción de la inversión y la promoción empresarial.

Para el caso de estudios sobre aglomeración y especialización económica, el de AndrésRosales y Villegas (2015) realiza un análisis sobre cómo el homicidio afecta estos comportamientos económicos. En lo particular, encuentran que la productividad es afectada en un $2 \%$ por la incidencia delictiva, imposibilitando con ello una completa consolidación de la actividad económica en diferentes partes del país. En una asociación derivada, por una parte, la investigación de Viridiana Ríos (2016) comprueba los efectos negativos que tiene la delincuencia en la diversificación económica, pues un aumento de hasta el $9.8 \%$ en los incidentes llega a extinguir un sector económico; y, por otra parte, el estudio de Padilla (2013) indica que para la ciudad de Culiacán, Sinaloa, la incidencia delictiva presente ha afectado gravemente varios indicadores de desempeño económico, como lo es la inversión extranjera directa (IED), para el periodo que comprende de 1999 a 2009.

Como se puede apreciar, cada uno de los anteriores trabajos estudia la afectación de la criminalidad de forma diferente, aunque todos ellos son posibles de considerar como referentes de casos de estudio. De esta manera, el presente trabajo se circunscribe a estos otros esfuerzos desarrollados en aras de evaluar en qué medida la delincuencia y la inseguridad llegan a afectar a las empresas y su localización. Lo anterior, enfocándose a los estados que comprenden la región centro-occidente de México: Aguascalientes, Michoacán, Nayarit, Jalisco, Colima y Guanajuato.

\section{Marco teórico}

En dónde se localizan y concentran las empresas ha sido un trabajo desarrollado particularmente en la teoría económica regional y espacial desde la perspectiva de los cuatro mercados que comprenden a un sistema económico (trabajo, vivienda, transporte, servicios públicos). La interacción de estos, al final, promueve la concentración de las actividades humanas y económicas (Krugman, 1998). No obstante, de entre estos, el mercado 
del trabajo es el considerado como el más relevante dada su relación con las actividades productivas y su concentración en las zonas urbanas (Rosenthal \& Ross, 2010).

Bajo esta arista, se busca entender el funcionamiento de una región o ciudad, y se basa en el supuesto de que las empresas se aglomeran en el territorio debido a la necesidad de obtener mayores ganancias que solo pueden ser obtenidas mediante la colaboración con otras unidades económicas relacionadas - economías de localización o MARo complementarias - de urbanización o Jacobianas - (Rosenthal \& Strange, 2004), así como por el entorno favorecedor que se genera tras dicha cooperación, como es la captación de mano de obra capacitada y la creación de sistemas productivos más eficientes (Camagni, 2005; Glaeser, 2010; Rosenthal \& Strange, 2004).

Sin embargo, un efecto contraproducente ocasionado por excesos en esta concentración o aglomeración económica (Marshall, 2010) de la actividad productiva es la creación de deseconomías causadas por externalidades negativas (Canback Samouel \& Price, 2006; Pichardo-Muñíz y Chavarría, 2016) que son causadas - entre otras como la congestión vial o la contaminación ambiental - por las actividades delictivas (Brueckner, 2011; O’Sullivan, 2012) que, para este caso en particular, son aquellas llevadas a cabo contra las empresas y de alto impacto social.

Por su parte, el debate que existe en la actualidad sobre qué son los "delitos contra las empresas" ha generado varios estudios sobre la victimización empresarial, definiciones conceptuales y herramientas diversas para su uso y análisis del fenómeno que tomaron como inicio la primera definición propuesta por Ewart y Tate (2007) para este tipo de delitos. Dentro de estos se consideran actualmente a aquellos que afectan a las unidades económicas tanto porque son de origen interno por empleados o dueños; o bien, de manera externa como criminales, agentes públicos o compradores, entre otros (Mugellini, 2013). En contraparte, los delitos de alto impacto social son más comunes en la literatura criminológica puesto que entre las ventajas que presentan está la mayor confiablidad que poseen como registros administrativos para reflejar puntualmente el fenómeno (Reynolds, 1980, p. 31). Entre ellos se encuentran el homicidio, el robo a transeúnte y el secuestro.

Más allá del debate criminológico sobre la composición de cada conjunto de delitos, desde una postura económica, una alta incidencia delictiva existente se traduce como un indicador de una pobre salud del sistema productivo analizado (Cook, 2009), por lo que se considera que tienen afectaciones negativas tanto en cuestiones de comportamiento económico - o directas — , así como de percepciones - o indirectas -

En lo particular, esto es relevante para la zona que comprenden a los estados del centrooccidente de México, pues en años recientes, y ocasionado entre otras cosas por la lucha entre cárteles del narcotráfico y contra las autoridades federales, que buscan erradicarlos 
mediante diversos operativos organizados desde la presidencia encabezada por Felipe Calderón (2006-2012), la incidencia delictiva presentada en varios municipios de los estados de Aguascalientes, Colima, Guanajuato, Jalisco, Michoacán y Nayarit ha ido considerablemente al alza, aun cuando se considera que a nivel nacional este fenómeno ha tomado la misma tendencia (Atuesta \& Ponce, 2016; ONc, 2017).

Esta región ha sido particularmente afectada debido a su alta capacidad productiva y su capacidad de especialización en el sector agrícola (Serrano, 2009) y manufacturero de la industria automotriz (El Financiero, 2017), así como por ser vía de comunicación terrestre con el resto del país y otros países.

\section{Métodos}

Para realizar este trabajo, y para ser usados como variable dependiente, se obtuvieron los datos de las unidades económicas a nivel municipal desagregados por especialización económica en tres grandes sectores: industria, comercio y servicios; por parte del Directorio Estadístico Nacional de Unidades Económicas (Denue) del Inegi para los años 2011 y 2015 (Inegi, 2017). Para poder aproximar los cambios presentados en cuanto a la localización de las empresas se realiza una diferencia de totales de 2015 respecto de 2011 ([delta_UE】_ij). Esta operación permite conocer qué zonas geográficas prefirieron las empresas para instalarse - o concentrarse - dado el periodo de tiempo de interés.

A su vez, esta variable fue desagregada en sectores derivados de su línea temática de actividad económica. Estas agrupaciones fueron obtenidas según lo estipulado por la Secretaría de Economía del Gobierno Federal de México, la cual se ve reflejado en el Denue (Inegi, 2017) en veinte subsectores y otras tantas más ramas o clases productivas. El objetivo de estas clasificaciones es poder hacer análisis en grandes agrupamientos de las empresas, aunque en lo específico estas sean diversas en su actividad. En este proceso se incluyen cuatro sectores: agrícola, servicios, comercio e industria. De los anteriores, solo se toman los tres últimos debido a que gran parte del sector agropecuario tiene una predisposición a localizarse en donde lo hace por condiciones específicas - como puede ser la factibilidad de la tierra para la siembra de granos específicos o la ubicación de las minas, por ejemplo - Derivado de lo anterior, el número de empresas analizadas no tendría por qué ser el mismo tanto a nivel agregado como en la clasificación sectorial, pues en los casos específicos las unidades económicas correspondientes a la producción agrícola no son cubiertos; no obstante, el número de observaciones analizadas se mantiene para todos los modelos planteados, dado que todos los municipios objeto de estudio contaron con al menos una unidad económica de los sectores productivos analizados. 
Por otra parte, como variables independientes se usan los registros administrativos de las carpetas de investigación levantadas por las procuradurías de justicia de cada entidad, recopilados por el Secretariado Ejecutivo del Sistema Nacional de Seguridad Pública (SESNSP, 2017). De entre estas carpetas, se usaron aquellas que cubrieran incidencia delictiva del fuero común, y se agruparon en dos conjuntos de variables: "delitos contra las empresas", que conjunta a aquellos contra el patrimonio: el robo común (a negocio y transportista) y el robo en carretera (a camiones de carga y a autobuses); así como los "delitos de alto impacto social", que se compuso de homicidios dolosos, secuestro y extorsión. Así como en el caso de las unidades económicas, los datos de incidencia delictiva fueron obtenidos de manera agregada para los años 2011 y 2015. Posterior a esto, se hace una diferencia entre los datos de 2011 y 2015 para inmediatamente después darles un tratamiento a los datos al relativizarlos por cada 10000 habitantes, según como es tradicional en la literatura criminológica.

Como datos control se imputa la inversión extranjera directa a nivel municipal obtenida del agregado estatal, se utiliza la educación promedio por habitantes mayores de edad $(E d u)$, la condición de urbanización del municipio de acuerdo con su población total para el año 2010 (Urbmetro), el total de elementos de la corporación policial adscritos a cada municipio (policía) y si los municipios recibieron algún tipo de apoyo gubernamental en materia de seguridad pública mediante subsidios o programas establecidos (SUBSEMUN y FASP).

Las herramientas analíticas usadas fueron la técnica de mínimos cuadrados ordinarios para ver los efectos generales. Para lograr dicho objetivo, el modelo propuesto queda planteado como se muestra en la fórmula 1:

$$
\begin{gathered}
\text { delta_UE } E_{i j}=\beta_{0}-\beta_{1} D C E_{i}-\beta_{2} D A I_{i}+\beta_{3} \text { Policia } * F A S P_{i} \\
\beta_{5} \text { Urbmetro }_{i}+\varepsilon_{i}
\end{gathered}
$$

Una versión que desagrega los delitos en lo particular queda descrita según la fórmula 2:

$$
\begin{aligned}
\text { delta }_{-} U E_{i j}= & \beta_{0}-\beta_{1} d p_{i}-\beta_{2} r c_{i}-\beta_{3} \text { renc }_{i}-\beta_{4} \text { hom }_{i}-\beta_{5} \text { Sec }_{i}-\beta_{6} \text { ext }_{i}+\beta_{7} \text { Policia } * \text { FASP }_{i}+ \\
& \beta_{8} \text { SUBSEMUN }_{i}+\beta_{9} I E D_{i}+\beta_{10} \text { Edu }_{i}+\beta_{11} \text { Urbmetro }_{i}+\varepsilon_{i}
\end{aligned}
$$

Para ambas fórmulas, la i representa el municipio analizado y la j es el sector económico analizado. De las variables explicativas usadas para la fórmula 1, DCE agrupa los así considerados delitos contra las empresas y los DAI a los delitos de alto impacto; en la segunda fórmula analítica, $d p$ se refiere a los delitos patrimoniales, $r c$ a robo común, renc a robo en carretera, hom a homicidios dolosos, sec a secuestros y ext a extorsiones. Como variables de control para ambos acercamientos por analizar se usan Policía que se refiere al número de policías locales por municipio, el FASP y SUBSEMUN como programas públicos 
de mejora para políticas de seguridad pública, IED como la inversión extranjera directa imputada a nivel municipal, $E d u$ como el nivel promedio educativo por municipio para la población mayor de edad y Urbmetro, que clasifica el grado de urbanización que presenta cada municipio según la población registrada con vivienda en cada municipio.

Por su parte, para particularizar en la relación por cada municipio, se utiliza el índice local de asociación espacial (LISA) bivariado, con el fin de observar los efectos de la concentración en el espacio de la incidencia delictiva (en delitos de alto impacto - DAI- y contra las empresas - DCE-) y el de la creación de nuevas empresas tanto a nivel global como local en conjuntos no estacionarios (Anselin, 1995).

\section{Resultados}

A nivel municipal, se observa en la figura 1 que, con relaciones al 95\% de significancia, la asociación espacial existente entre la creación de nuevas empresas - variable $1-\mathrm{y}$ los delitos contra las empresas (alto-alto) se concentra desfavorablemente en el estado de Jalisco, pues hay alta incidencia delictiva; por el contrario, en municipios de Guanajuato y la zona costera de Michoacán se observa que hay baja criminalidad, pero alta creación de empresas (alto-bajo). El caso más grave según este análisis se encuentra en gran parte de los municipios de Jalisco, en los que denota una poca creación de nuevas empresas y una alta incidencia delictiva (bajo-alto). No obstante, cuando se analiza la asociación global para la región, la relación entre la creación de nuevas empresas y los delitos contra las mismas es generalizado pues la I de Morán calculada a 99 permutaciones resulta en un valor global de 0.0400 .

Por otra parte, cuando se hace referencia a la asociación entre la creación de empresas y los delitos de alto impacto reportados ante las autoridades competentes, la asociación global vista en la figura 2 reporta un estadístico de Morán no significativo al 95 \% ( $I$ de 0.0299, pseudo $\mathrm{p}=0.07)$ que refleja una aún mayor dispersión de los fenómenos analizados para todo el territorio de interés.

No obstante, el patrón observado con los delitos contra las empresas se repite en gran medida cuando se consideran los delitos de alto impacto. Para el periodo analizado, Guanajuato es una entidad con alta creación de empresas y baja incidencia delictiva que contrasta con Jalisco, pues este segundo estado presenta casi exclusivamente a los municipios con baja actividad económica, pero alta incidencia delictiva.

Analizando los efectos por sector económico, según se muestra de la tabla 1, en lo general se puede decir que no hay una afectación directa de agrupar los delitos. No obstante, si se observan los resultados por sector, hay algunos resultados llamativos. Uno de ellos es que el sector industrial responde negativamente ante la presencia de los delitos de 
alto impacto (M2) y, en contraparte, el sector comercial lo hace ante los delitos contra las empresas (M4).

Este hecho iría a favor de la hipótesis planteada al inicio de este trabajo sobre efectos negativos en la localización económica ocasionada por la incidencia delictiva. Empero, solo para el sector comercial, se logra corroborar la idea de una manera efectiva, dado que es significativa al 95\%. Para el sector servicios, ambas agrupaciones de delitos resultan negativas, aunque sin posibilidad de realizar inferencia con los estimadores obtenidos.

Guando se hace una revisión de la afectación de los delitos en lo particular, según lo que se obtiene de la tabla 1, solo para el sector comercial resultan los delitos patrimoniales significativos y acordes al signo esperado (M4a). Para el resto de los delitos, si bien hay casos significativos para el sector industrial, estos resultan con signos contrarios a lo planteado en los modelos teóricos. Los otros delitos, sean agregados o desagregados, no resultan significativos.

Figura 1. Autocorrelación espacial bivariada de unidades económicas vs. delitos contra las empresas (DCE).

\section{Clústeres de nuevas empresas y DCE}

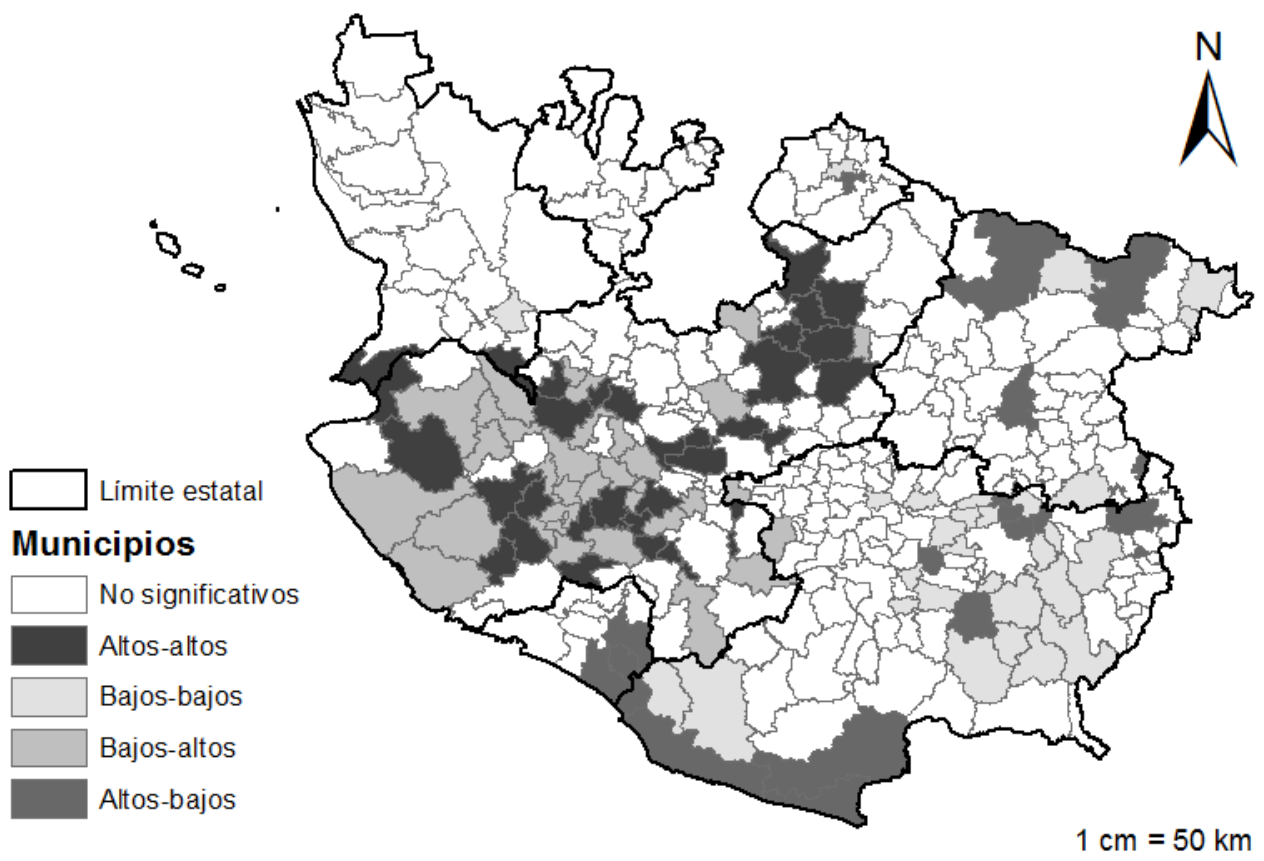

Figura 2. Autocorrelación espacial bivariada de unidades económicas y DAI. 
Fuente de ambas: elaboración propia con datos del Denue y el SESNSP.

\section{Clústeres de nuevas empresas y DAI}

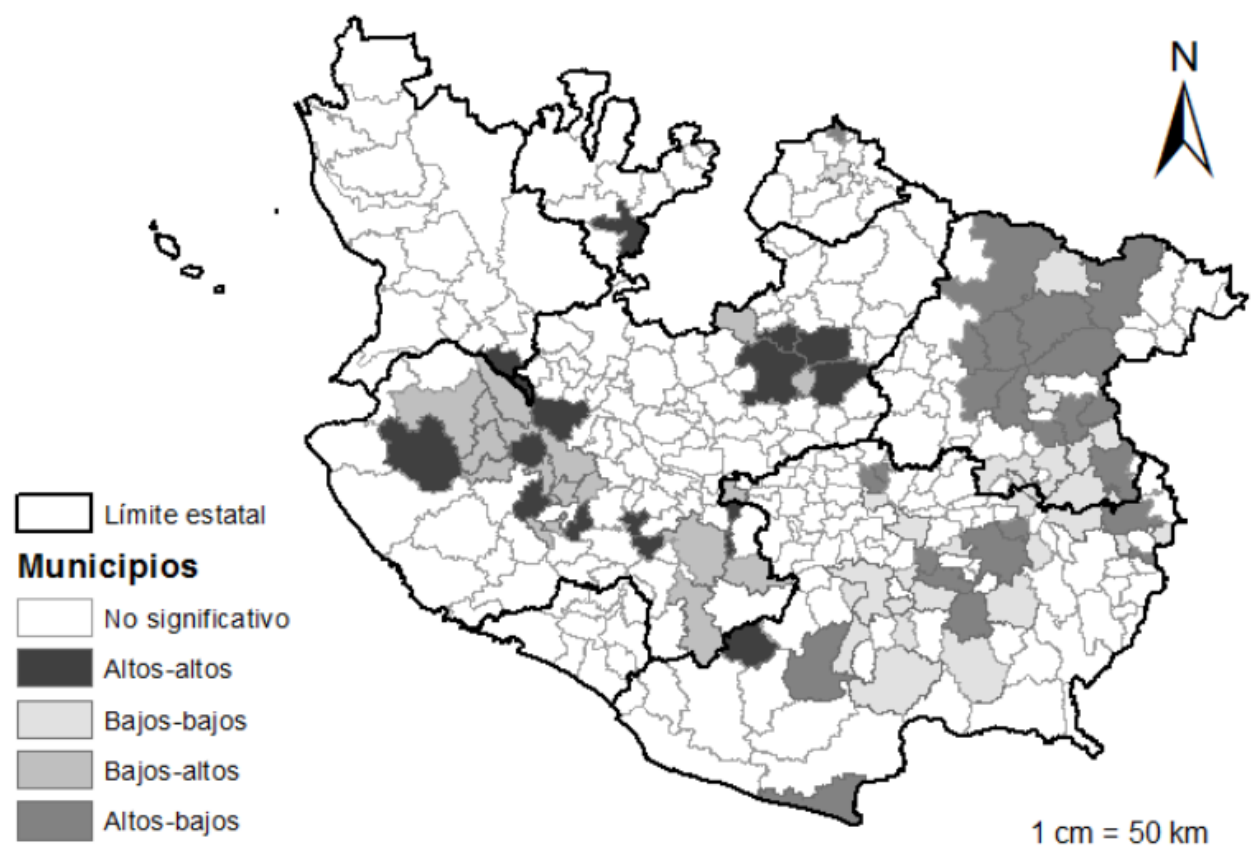

Tomando como referencia el resto de las variables usadas como control, se encuentra que para los modelos generales ( 1 y mla) todas las variables de caracterización social y económicas resultan significativas y acordes a la teoría, así como a la hipótesis planteada en la fórmula del modelo. Lo anterior no sucede igualmente con las variables que responden a subsidios para fortalecer la capacidad municipal en materia de seguridad pública o bien para la cantidad de elementos con los que cuentan las corporaciones locales. De entre estas variables, solo es la referente al nivel educativo la que mantiene una capacidad explicativa que se traslada a casos particulares, como son el sector comercio y el de servicios.

\section{Conclusiones}

La relación existente entre la economía y el crimen es intrigante, pues no necesariamente sigue lo propuesto por diversas teorías asociadas al tema. Por otra parte, la importancia que tiene un Estado de derecho en materia de seguridad pública cada vez se vuelve más relevante al ser considerado como un factor clave para promover la estabilidad, 
el crecimiento sostenido y la competitividad de un Estado (Fajnyzlber, Lederman, \& Loayza, 2001; Pichardo-Muñiz, \& Chavarría, 2016).

Dado el clima de alza que ha registrado la incidencia delictiva en todo el territorio nacional mexicano, durante buena parte del siglo xxi los trabajos académicos enfocados a detallar la interacción presentada en la relación delincuencia-economía han florecido. Casos como estos son los previamente mencionados de Ríos (2016), Sloan et al. (2016) o Andrés-Rosales y Villegas (2015), en los que se analizan cuestiones como la especialización económica, la afectación a un sector productivo en específico o en dónde se localizan unidades económicas que corresponden al sector de servicios como son los restaurantes. La idea planteada en este trabajo busca complementar los anteriores estudios, pero enfatizando para un caso específico en el territorio de México.

Si bien de los resultados del ejercicio econométrico no se obtienen estimaciones de mucha relevancia, se puede resaltar que efectivamente, y según como se ha planteado en trabajos previos, los sectores económicos no son solo afectados presumiblemente, sino que también responden perceptualmente de forma diferente a delitos que no les afectan de manera directa - o en su productividad-, como son los delitos de alto impacto. No obstante la aparente debilidad estadística de los resultados, estos pueden deberse a que los análisis se hicieron en un nivel macroagregado; por lo anterior, los efectos encontrados podrían verse resaltados al analizarse en lo particular por subsectores y ramas, incorporando así efectos intra e intersectoriales que con los modelos actuales pudieran no haber sido captados correctamente por mínimos cuadrados ordinarios.

De considerar este estudio como una antesala y realizar ejercicios con mayor rigor o especificidad regional, los resultados anteriores pueden ser considerados para la creación de políticas públicas de promoción específica a la actividad económica, desarrollo regional basado en economías de aglomeración y atenciones de seguridad pública que se enfoquen en delitos específicos que atentan contra las unidades económicas, como lo mencionan Matti y Ross (2016) o Rosenthal y Ross (2010). Por otra parte, la labor realizada en este trabajo es factible de ser mejorada en términos metodológicos y estadísticos al considerar la aplicación de otras técnicas más apropiadas, según la disponibilidad y adecuación de la información. 
Revista CIFE / ISSN: 0124-3551 e-ISSN: 2248-4914 / Bogotá-Colombia / Vol. 21 N. 35 / julio-diciembre 2019 / pp. 45-59

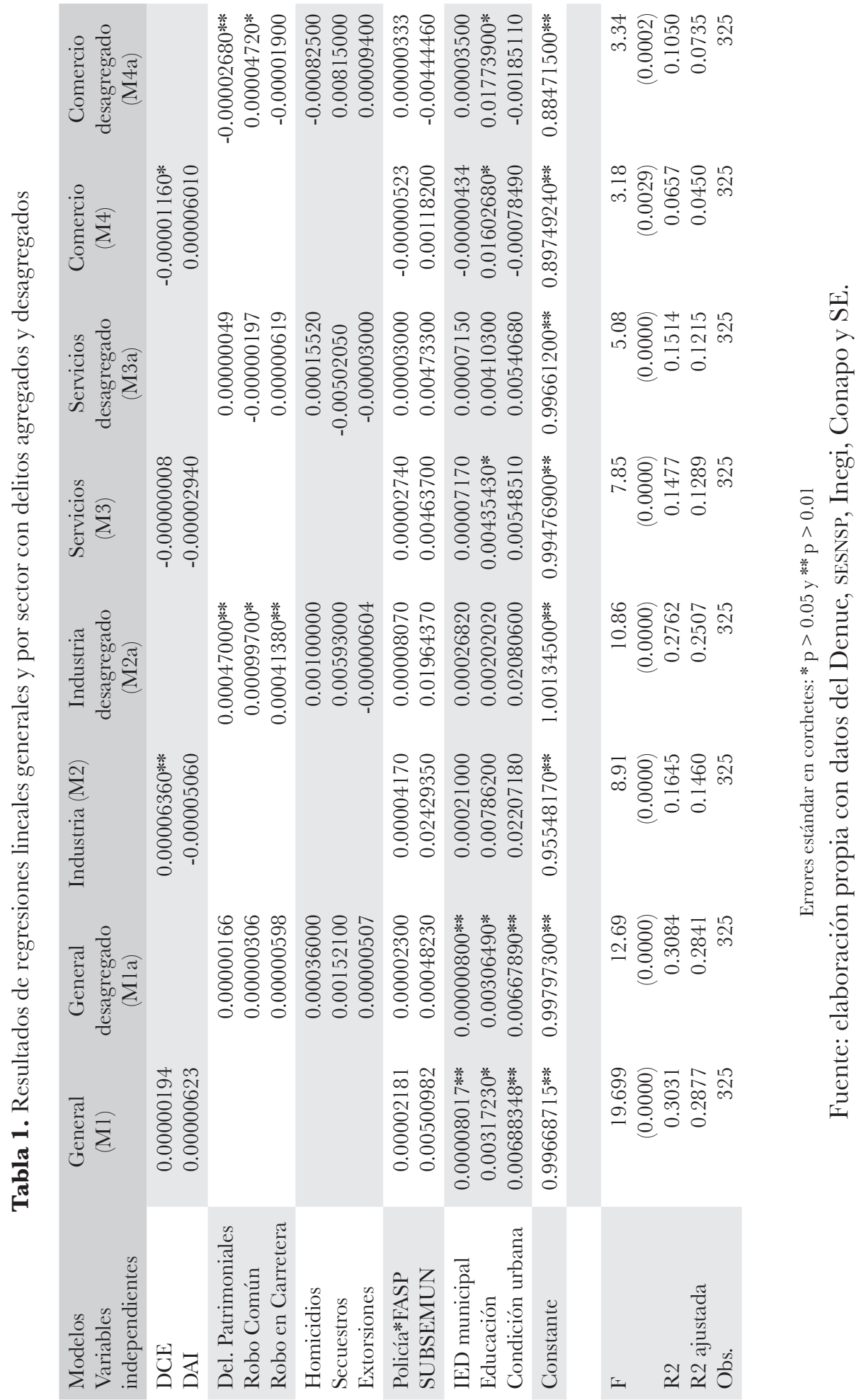




\section{Referencias bibliográficas}

Andrés-Rosales, R., \& Villegas, M. (2015). La inseguridad y el clúster automotriz en las regiones mexicanas: un análisis con datos de panel espacial, 1999-2009. Paradigma económico, 7(2) (jul.-dic.), 7-26.

Anselin, L. (1995). Local Indicators of Spatial Association-LISA. Geographical Analysis, 27(2), 93-115.

Atuesta, L., \& Ponce, A. (2016). Cómo las intervenciones de las fuerzas públicas de seguridad alteran la violencia. Evidencia del caso mexicano (Cuaderno de Trabajo, núm. 19). Programa de Política de Drogas, Centro de Investigación y Docencia Económicas, Región Centro. Aguascalientes, México. Recuperado de http://ppd. cide.edu/documents/302668/0/19_Intervenci\%C3\%B3nFINAL1.pdf

Brueckner, J. K. (2011a). Crime. En J. K. Brueckner (Ed.), Lectures on Urban Economics (pp. 207-230). Cambridge, Estados Unidos: Massachusetts Institute of Technology Press.

Camagni, R. (2005). Economía urbana. Barcelona, España: Antoni Bosch.

Canback, S., Samouel, P., \&. Price, D. (2006). Do diseconomies of scale impact firm size and performance? A Theoretical and empirical overview. Fournal of Managerial Economics, 4(1), 27-70.

Cook, P. J. (2009). "Crime in the city". En R. P. Inman (Ed.), Making Cities Work: Prospects and Policies for Urban America (pp. 297-327). Princeton, Estados Unidos: Princeton University Press.

Detotto, G., \& Otranto, E. (2010). Does Crime Affect Economic Growth? Kyklos, 63(3), 330-345.

El Financiero (30 de enero de 2017). Aguascalientes y Guanajuato, con mayor repunte económico. El Financiero. Recuperado de http:www.elfinanciero.com.mx

Enamorado, T., López-Calva, L. F., \& Rodríguez-Castelán, C. (2014). Crime and growth convergence: Evidence from Mexico. Economic Letters, 125(1), 9-13.

Ewart, B. W., \& Tate, A. (2007). Policing retail crime: from minor offending to organized criminal networks. En K. T. Froeling (Ed.), Criminology research focus (pp. 33-67). Nueva York, Estados Unidos: Nova Science Publishers.

Fajnzylber, P., Lederman, D., \& Loayza, N. (2001). Crimen y victimización: una perspectiva económica. En P. Fajnzylber, D. Lederman y N. Loayza (Eds.), Crimen y violencia 


\section{"Čl'F́'E 35}

Revista CIFE / ISSN: 0124-3551 e-ISSN: 2248-4914 / Bogotá-Colombia / Vol. 21 N.o 35 / julio-diciembre 2019 / pp. $45-59$ en América Latina (pp. 1-62). Washington D.C., Estados Unidos: Banco Mundial y Alfaomega.

Glaeser, E. L. (2010). Introduction. En E. L. Glaeser (Ed.), Agglomeration Economies (pp. 1-14). Chicago, Estados Unidos: The University of Chicago Press.

González-Andrade, S. (2014). Criminalidad y crecimiento económico regional en México. Frontera Norte, 26(51), 75-111.

Goulas, E., \& Zervoyianni, A. (2015). Economic growth and crime: is there an asymmetric relationship? Economic Modelling, 49, pp. 286-295.

Hamley, D. D., \& L. R. McPheters (1975). Crime as an externality of economic growth: An empirical analysis. The American Economist, 19(1), pp. 45-47.

IMCO (2007). Seguridad y competitividad: Efectos de cambios en variables institucionales. Ciudad de México: México, Instituto Mexicano para la Competitividad. Recuperado de http://imco.org.mx/wp-content/uploads/2007/12/seguridad_y_competitividad_ reformas_necesarias_06.pdf

Inegi (2017). Directorio Estadístico Nacional de Unidades Económicas (Denue). Aguascalientes, México: Instituto Nacional de Estadística y Geografía. Recuperado de http://www. beta.inegi.org.mx/app/mapa/denue/default.aspx

Inegi (2016). Encuesta Nacional de Victimización de Empresas 2016 ENVE. Marco conceptual. Aguascalientes, México: Instituto Nacional de Estadística y Geografía. Recuperado de http://internet.contenidos.inegi.org.mx/contenidos/Productos/prod_serv/contenidos/espanol/bvinegi/productos/nueva_estruc/702825089771.pdf

Krugman, P. (1998). What's new about the New Economic Geography. Oxford Review of Economic Policy, 14(2), 7-17.

Matti, J., \& Ross, A. (2016). Does crime affect entrepreneurship? A discussion of the current literature. Journal of Entrepreneurship and Public Policy, 5(3), 254-272.

Marshall, A. (1920). Principles of economics: An introductory volume (8th Ed.). Londres, Inglaterra: Macmillan.

Mendoza, V. (2014). Los 5 enemigos de la productividad en México. Forbes. Recuperado el 15 de febrero de 2017 de https://www.forbes.com.mx/ los-5-enemigos-de-la-productividad-en-mexico. 
Mugellini, G. (Ed.) (2013). Measuring and analyzing crime against the private sector: International experiences and the Mexican practice. Ciudad de México, México: Instituto Nacional de Estadística y Geografía.

ONC (2017). Reporte sobre delitos de alto impacto. Ciudad de México, México: Observatorio Nacional Ciudadano. Recuperado de http:/ / onc.org.mx/wp-content/ uploads/2017/05/170501mensual-marzo_2017_VF.pdf

O’ Sullivan, A. (2012). “Crime”. En A. O’Sullivan, Urban Economics (pp. 338-366). Nueva York, Estados Unidos: Mc Graw Hill.

Padilla, I. (2013). Economía y violencia urbana en Culiacán. Victimización y percepción de inseguridad en mipymes (Tesis de doctorado en Estudios Regionales, con énfasis en América del Norte). Facultad de Estudios Internacionales y Políticas Públicas, Universidad Autónoma de Sinaloa, Culiacán, México.

Pichardo-Muñiz, A., \& Chavarría, M. O. (2016). Agglomeration Economies Versus Urban Diseconomies: The Case of the Greater Metropolitan Area (GMA) of Costa Rica. En J. Burian, Advances in Spatial Planning (pp. 287-310). Estados Unidos: InTech.

Reynolds, M. O. (1980). "The Economics of Criminal Activity". En R. Andreano y J. J. Siegfried (Eds.), The Economics of Crime (pp. 27-70). Cambridge, Estados Unidos: John Wiley and Sons.

Ríos, V. (2016). The impact of crime and violence on economic sector diversity. Documento de trabajo. Harvard University, Boston, Estados Unidos. Recuperado de https://scholar.harvard.edu/vrios/publications/ impact-crime-and-violence-economic-sector-diversity

Rosenthal, S. S., \& Ross, A. (2010). Violent crime, entrepreneurship, and cities. Fournal of Urban Economics, 67(1), 135-149.

Rosenthal, S. S., \& Strange, W. C. (2004). Evidence on the Nature and Sources of Agglomeration Economies. En J. V. Henderson y J. F. Thiese (Eds.), Handbook of Regional and Urban Economics (Vol. 4) (pp. 2129-2167). Elsevier.

SESNSP (2017), Secretariado Ejecutivo del Sistema Nacional de Seguridad Pública. Incidencia Delictiva del Fuero Común. Ciudad de México, México: SESNSP. Recuperado de http://secretariadoejecutivo.gob.mx/incidencia-delictiva/incidencia-delictivafuero-comun.php

Serrano, M. (2009). Drug Trafficking and the State in Mexico. En H. R. Friman (Ed.), Crime and the Global Political Economy (pp. 139-158). Boulder, Estados Unidos: Lynne Rienner. 
Sloan, C., Cuadill, S. B., \& Mixon, F. G. (2016). Entrepreneurship and crime: The case of new restaurant location decisions. Fournal of Business Venturing Insights, 5, 19-26. 